\title{
Research on Numerical Integration Algorithm in Molecular Dynamics Simulation
}

\author{
Zijing Gao ${ }^{1, a}$ \\ ${ }^{1}$ School of China Agricultural University, Beijing 510000, China \\ a2328247224@qq.com
}

\begin{abstract}
Molecular dynamics is a combination of physics, mathematics and chemical synthesis technology. Molecular dynamics method is a computer simulation experimental method, which is a powerful tool for studying condensed matter system. This paper mainly introduces the development of molecular dynamics simulation, studies its motion law at the atomic (molecular) level, and briefly introduces the basic theory of molecular dynamics and the description of different simulation methods from macroscopic to microstructure. In this paper, we study the basis of different theoretical algorithms in numerical integration. We integrate the numerical integration algorithms such as Verlet method, Leap-frog method, Velocity Verlet method, Beeman method and so on and we are dedicated to study the accuracy, both advantages and disadvantages of these algorithms, hoping to be able to improve and develop new numerical integration algorithms on this basis.
\end{abstract}

Keywords: Molecular dynamics, Finite difference method, Verlet method, leap-frog method, Velocity Verlet method

\section{Introduction}

The molecular dynamics simulation refers to the calculation of the interaction and motion between the microscopic particles in the multibody system formed by the nucleus and the electron. Also, each nucleon is regarded as such a kind of movement under Newton's law by the action of the potential field composed of all the other nuclei and electrons. And then we can get the motion trajectory of the particles in the system, and calculate the macroscopic properties of the structure and properties of the material according to the statistical physical method.

In 1957, Alder and Wainwright [1] first used molecular dynamics to study the state equations of gases and liquids under the hard sphere model, thus pioneering the use of molecular dynamics simulations to study the macroscopic properties of matter. Later, people have made a lot of improvements to this method, a lot of researches had been done by exerting its defects to the solid and liquid. However, due to the speed of the computer and memory constraints, early simulation of the spatial scale and time scale are subject to great restrictions. In the late 1980s, due to the rapid development of computer technology, coupled with the development of multi-body potential function, molecular dynamics simulation technology has been injected new vitality.

The basic intention of molecular dynamics simulations is to reproduce the real process of nature on the computer, which includes the processes that have actually occurred, as well as the process in which the experimental conditions are not yet permitted. This requires that the more real the potential function is, the better the basic intention of molecular dynamics simulations are, otherwise it will bring the error. However, molecular dynamics simulations are also particularly suited to achieving "thought experiments" that do not have to be quantitatively consistent with laboratory experiments but that can characterize or confirm some qualitative conclusions [2 5].

In view of the large amount of budgets and some physical quantities that are not easy to be solved with the integral solution, we have to develop numerical computational summation methods at different molecular levels: molecular dynamics (MD) method [6] and Monte Carlo (MC) method [7]. These two methods can be used to calculate the macroscopic physical quantity according to the arrangement of molecules and motion. In the microscopic case, we can solve some empirical models by using the dissimilar particle dissipative studies of polymer hydrodynamics [8] or using the 
Schrodinger equation [9]. However, due to the strong theoretical, smaller budget range and excessive computational complexity of the Schrodinger equation, we have developed some relatively rough numerical integration algorithms [10] with the "computer experiment" to establish a theoretical and experimental bridge. Fortunately, we still can achieve good accuracy and provide a more accurate microstructure, particle movement and their relationship with the macroscopic nature of the material clear images.

\section{The Basic Content of Molecular Dynamics Method}

\subsection{Basics}

Calculate the phase space of a group of molecules, each of which subordinates Newton's law of motion.

\subsection{Molecular Dynamics Method}

Molecular dynamics is not only an important computer simulation method for solving multi-body problems at the atomic and molecular levels, but also a significant method to predict the dynamic properties of nanoscale materials; By solving the motion equations of all particles, the molecular dynamics method can be used to simulate the basic process associated with the atomic motion path; In molecular dynamics, the motion behavior of particles is described by the classical Newtonian equations of motion; The molecular dynamics method is a deterministic method, and once the initial configuration and velocity are determined, the trajectories of the molecules produced over time are determined, too.

\subsection{Molecular Dynamics Method}

In principle, the microchemical system to which the molecular dynamics method is applied is not limited. This method can be applied to the few-body system as well as multi-body system; It can be either a point particle system or a system with an internal structure; The microscopic object of treatment can be either a molecule or other microscopic particles. Since the mid-1950s, molecular dynamics has been widely used. It has become an important method of computer simulation with the Monte Carlo method. Many important results have been achieved by molecular dynamics, such as the state equation of gas or liquid, the phase change problem, the adsorption problem, and the non-equilibrium process.

\section{Motion Equations of Molecular Dynamics}

The main object of molecular dynamics research is a series of molecules. Assuming that the number of molecules studied is $N$, the Newtonian equation of motion to describe the motion of the molecule, that is: $m_{i} d^{2} r_{i} / d t^{2}=-\nabla_{i} V\left(r_{1}, r_{2}, r_{N}\right)$, where $m_{i}, \quad r_{i}$ are respectively the $\mathrm{i}^{\text {th }}$ atom of the quality and location. $\nabla_{i}=-\partial / \partial r_{i}, V\left(r_{1}, r_{2}, r_{N}\right)$ (the potential energy system).

\section{Numerical Integration of Equations of Motion}

By using the molecular mechanics force field, it is assumed that any atomic motion in the system conforms to the classical Newtonian mechanics equation. When the initial coordinates and velocities are given, the trajectory of each atom can be obtained after integration. The basic point of the computer simulation method is to use the advantages of high speed and precision of the modern computer, in order to numerically calculate the motion equations of hundreds of, even thousands of molecules. At present, many different integration methods have been reported. They are different in efficiency and accuracy, and the finite difference method is used to integrate the two order ordinary differential equations, which are commonly put into application, such as Verlet method, Leap-frog method, Velocity Verlet method, Beeman's method, Gear method, and so on.

\subsection{Basics: Finite Difference Techniques}

It is assumed that the position and dynamics of the system can be expanded by Taylor series: 


$$
r(t+\Delta t)=r(t)+\frac{d r(t)}{d t} \Delta t+\frac{1}{2 !} \cdot \frac{d^{2} r(t)}{d t^{2}}(\Delta t)^{2}+\frac{1}{3 !} \cdot \frac{d^{3} r(t)}{d t^{3}}(\Delta t)^{3}+\frac{1}{4 !} \cdot \frac{d^{4} r(t)}{d t^{4}}(\Delta t)^{4}+\ldots
$$

Based on kinetic properties:

$$
\begin{gathered}
r(t+\Delta t)=r(t)+v(t) \Delta t+\frac{1}{2 !} \cdot a(t)(\Delta t)^{2}+\frac{1}{3 !} \cdot b(t)(\Delta t)^{3}+\frac{1}{4 !} \cdot c(t)(\Delta t)^{4}+\ldots \\
v(t+\Delta t)=v(t)+a(t) \Delta t+\frac{1}{2 !} \cdot b(t)(\Delta t)^{2}+\frac{1}{3 !} \cdot c(t)(\Delta t)^{3}+\ldots \\
a(t+\Delta t)=a(t)+b(t) \Delta t+\frac{1}{2 !} \cdot c(t)(\Delta t)^{2}+\ldots
\end{gathered}
$$

$r$ is the position of the particle, $v$ is the particle velocity, $a$ is the particle acceleration, $t$ is the simulation step.

\subsection{Verlet Method}

Based on the idea of finite difference method:

(4) $+(5)$ :

$$
\begin{aligned}
& r(t+\Delta t)=r(t)+v(t) \Delta t+\frac{1}{2 !} \cdot a(t)(\Delta t)^{2}+\frac{1}{3 !} \cdot b(t)(\Delta t)^{3}+\frac{1}{4 !} \cdot c(t)(\Delta t)^{4}+\ldots \\
& r(t-\Delta t)=r(t)-v(t) \Delta t+\frac{1}{2 !} \cdot a(t)(\Delta t)^{2}-\frac{1}{3 !} \cdot b(t)(\Delta t)^{3}+\frac{1}{4 !} \cdot c(t)(\Delta t)^{4}+\ldots
\end{aligned}
$$

$$
r(\mathrm{t}+\Delta \mathrm{t}) \approx 2 r(\mathrm{t})-\mathrm{r}(\mathrm{t}-\Delta \mathrm{t})+a(t)\left(\Delta \mathrm{t}^{2}\right)+o\left(\Delta \mathrm{t}^{4}\right)
$$

Which $a(t)$ can be obtained by potential energy:

According to the mean value theorem:

$$
a(t)=\frac{F}{m}=-\frac{1}{m} \cdot \frac{\partial V}{\partial r}
$$

$$
v(\mathrm{t})=\frac{[r(t+\Delta t)-r(t-\Delta t)]}{2 \Delta t}
$$

Advantages: this method is more accurate. We conclude that the error is $o\left(\Delta t^{4}\right)$ from (6); Only one force is calculated for each integral; Time is relatively reversible.

Disadvantages: $2 r(\mathrm{t})$ and $r(t-\Delta t)$ are significantly large and $a(t)\left(\Delta \mathrm{t}^{2}\right)$ is pretty small. The addition can cause loss of accuracy; The error of $v(\mathrm{t})$ is $o\left(\Delta \mathrm{t}^{2}\right)$ and is relatively bigger compared to the error of $r(t)$. There is only the location of the time evolution data. Also, the speed needs to be obtained from the location, which is very awkward processing speed.

\subsection{Leap-frog Method}

$$
\begin{aligned}
& v\left(t+\frac{\Delta t}{2}\right)=v(t)+a(t) \frac{\Delta t}{2}+\frac{1}{2 !} b(t)\left(\frac{\Delta t}{2}\right)^{2}+\ldots \\
& v\left(t-\frac{\Delta t}{2}\right)=v(t)-a(t) \frac{\Delta t}{2}+\frac{1}{2 !} b(t)\left(\frac{\Delta t}{2}\right)^{2}-\ldots
\end{aligned}
$$

(7) - (8):

$$
\begin{gathered}
v\left(t+\frac{\Delta t}{2}\right)=v\left(t-\frac{\Delta t}{2}\right)+\mathrm{a}(t) \Delta t+o\left(\Delta t^{3}\right) \\
r\left(t+\frac{\Delta t}{2}+\frac{\Delta t}{2}\right)=r\left(t+\frac{\Delta t}{2}\right)+\mathrm{v}\left(t+\frac{\Delta t}{2}\right) \frac{\Delta t}{2}+\frac{1}{2 !} a\left(t+\frac{\Delta t}{2}\right)\left(\frac{\Delta t}{2}\right)^{2}+\ldots
\end{gathered}
$$




$$
r\left(t+\frac{\Delta t}{2}-\frac{\Delta t}{2}\right)=r\left(t+\frac{\Delta t}{2}\right)-\mathrm{v}\left(t+\frac{\Delta t}{2}\right) \frac{\Delta t}{2}+\frac{1}{2 !} a\left(t+\frac{\Delta t}{2}\right)\left(\frac{\Delta t}{2}\right)^{2}+\ldots
$$

(9) - (10):

$$
r(t+\Delta t)=r(t)+\mathrm{v}\left(t+\frac{\Delta t}{2}\right) \Delta t+o\left(\Delta t^{3}\right)
$$

Advantages: Including the apparent velocity; Convergence speed is very fast, the calculation is small; Trajectory is related to the speed; Position, speed error are both the $o\left(\Delta t^{3}\right)$.

Disadvantages: The speed and the coordinates are not synchronized in time.

\subsection{Velocity Verlet Method}

In order to obtain the same time position and speed, and at the same time we can make the position, speed error continue to shrink or remain unchanged.

$b(t)$ can be solved from (3), then brought into (2):

$$
\begin{gathered}
r(t+\Delta t)=r(t)+v(t) \Delta t+\frac{1}{2} a(t) \Delta t^{2}+o\left(\Delta t^{3}\right) \\
v(t+\Delta t)=v(t)+\frac{1}{2}[a(t)+a(t+\Delta t)] \Delta t+o\left(\Delta t^{3}\right)
\end{gathered}
$$

Advantages: Speed calculation is more accurate, whose method can give the location, speed and acceleration at the same time without sacrificing accuracy. Also, this method gives the apparent velocity. The calculation is moderate and the application is of a wide range currently.

\subsection{Beeman's Method}

In order to improve the accuracy further, according to (3), we get:

$$
a(t-\Delta t)=a(t)-b(t) \Delta t+\frac{1}{2 !} \cdot c(t)(\Delta t)^{2}+\ldots
$$

Then we can solve $b(t)$ and $c(t)$.

$$
\begin{gathered}
r(t+\Delta t)=r(t)+v(t) \Delta t+\left[\frac{2}{3} a(t)-\frac{1}{6} a(t-\Delta t)\right](\Delta t)^{2} \\
v(t+\Delta t)=v(t)+\frac{a(t+\Delta t) \Delta t}{3}+\frac{5 a(t)-a(t-\Delta t)}{6} \Delta t
\end{gathered}
$$

Advantages: This approach counteracts its complexity, and due to the demand of maintaining the "old" acceleration methods traditionally. Also, this method improves its accuracy, more accurate than the Verlet method, and achieves better in terms of energy conservation.

\subsection{Gear Method}

In 1971, Gear proposed a Gear algorithm based on the predictive-corrected integration method. The algorithm can select different highest order differentials, the correction factor order, according to different calculation requirements, so that the series of truncation errors can be adjusted appropriately.

$$
\left.\begin{array}{l}
r^{p}(t+\Delta t)=r(t)+v(t) \Delta t+\frac{1}{2} a(t)(\Delta t)^{2}+\frac{1}{6}(\Delta t)^{3} b(t)+\ldots \\
v^{p}(t+\Delta t)=v(t)+a(t) \Delta t+\frac{1}{2} b(t)(\Delta t)^{2}+\ldots \\
a^{p}(t+\Delta t)=a(t)+b(t) \Delta t+\ldots \\
b^{p}(t+\Delta t)=b(t)+\ldots
\end{array}\right\}
$$

A set of vectors is defined by the above, where $r_{0}$ is the position of the atom.

$$
r_{1}=v_{0} \Delta t
$$




$$
\begin{aligned}
& r_{2}=\frac{1}{2} a_{0} \Delta t^{2} \\
& r_{3}=\frac{1}{6} b_{0} \Delta t^{3}
\end{aligned}
$$

The above vectors satisfy the following relationship:

$$
\left(\begin{array}{l}
r_{0}^{p}(t+\Delta t) \\
r_{1}^{p}(t+\Delta t) \\
r_{2}^{p}(t+\Delta t) \\
r_{3}^{p}(t+\Delta t)
\end{array}\right)=\left(\begin{array}{llll}
1 & 1 & 1 & 1 \\
0 & 1 & 2 & 3 \\
0 & 0 & 1 & 3 \\
0 & 0 & 0 & 1
\end{array}\right)\left(\begin{array}{c}
r_{0}(t) \\
r_{1}(t) \\
r_{2}(t) \\
r_{3}(t)
\end{array}\right)
$$

The correction factor that the Gear algorithm predicts is:

$$
\left(\begin{array}{l}
r_{0}^{c}(t+\Delta t) \\
r_{1}^{c}(t+\Delta t) \\
r_{2}^{c}(t+\Delta t) \\
r_{3}^{c}(t+\Delta t)
\end{array}\right)=\left(\begin{array}{l}
r_{0}^{p}(t+\Delta t) \\
r_{1}^{p}(t+\Delta t) \\
r_{2}^{p}(t+\Delta t) \\
r_{3}^{p}(t+\Delta t)
\end{array}\right)+\left(\begin{array}{c}
c_{0} \\
c_{1} \\
c_{2} \\
c_{3}
\end{array}\right) \Delta r
$$

\section{Conclusions}

At present, in engineering application and scientific research, the computer numerical simulation method has gradually become an important approach to solve complex problems. With the change of the times, the method of numerical integration algorithm in molecular dynamics simulation is more and more accurate and avoids the previous shortcomings. After continuous improvement and perfection, the position, velocity and other data of the particle which are at the given moment is not only improved with the accuracy, but also gradually achieved to choose a different degree of corresponding numerical integration method according to people's needs. Meanwhile, improving the accuracy and significant improvement in energy conservation can be achieved. I believe we can improve the accuracy, at the same time, to ensure that the amount of calculation is within the acceptable range through the more rigorous calculation and the further refinement of the related algorithm.

\section{References}

[1] B.J. Alder, T.E. Wainwright. Studies in molecular dynamics. I. General Method [J]. Journal of Chemical Physics, 1959, 31(2):459-466.

[2] Y. Wen. An overview on molecular dynamics simulation [M]. Advances in Mechanics, 2003.

[3] Z.S Li, Y.J. Zhao, X.N. Jia, et al. Progress in computer simulation of molecular dynamics [J]. Mechanical Management Development, 2008, 23(2):174-176.

[4] W.C. Tian, Y.T. Yang. Molecular dynamics analysis of "Stiction" based on multi-body EAM potential function [J]. Journal of Computational \& Theoretical Nanoscience, 2013, 10(5):1252-1256. [5] T. Hansson, C. Oostenbrink, W.V. Gunsteren. Molecular dynamics simulations [J]. Current Opinion in Structural Biology, 2002, 12(2):190-196.

[6] H.X. Li, H.F. Qiang, G. Wang, et al. Simulation of diffusion behavior of plasticizer based on MD method [J]. 2009, 17(1):36-41.

[7] Z.G. Fang. Computer simulation and Monte Carlo method [M]. Beijing Institute of Technology Press, 1988.

[8] J.J. Zhou. Application of dissipative particle dynamics and navier boundary conditions in simulating nano-sized fluid dynamics [J]. Acta Polymerica Sinica, 2016(8):1021-1029.

[9] P.A. Ruprecht, M.J. Holland, K. Burnett, et al. Time-dependent solution of the nonlinear Schrodinger equation for Bose-condensed trapped neutral atoms. [J]. Physical Review A Atomic Molecular \& Optical Physics, 1995, 51(6):4704. 
[10] Z.F. Tong, C. Xiao, Z.L. Wei. Molecular dynamics simulation and its application in metallurgical slag [J]. Nonferrous Metals Science and Engineering, 2016, 7(3):15-20. 